\title{
Development of graphene oxide-wrapped gold nanorods as robust nanoplatform for ultrafast near-infrared SERS bioimaging
}

This article was published in the following Dove Press journal:

International Journal of Nanomedicine

9 June 2017

Number of times this article has been viewed

\author{
Xuejun Qiu,' Xinru You, ${ }^{2}$ \\ Xing Chen, ${ }^{2}$ Haolin Chen, ${ }^{1}$ \\ Arvind Dhinakar, ${ }^{3}$ Songhao \\ Liu,' Zhouyi Guo,' Jun \\ Wu, ${ }^{2,4}$ Zhiming Liu' \\ 'SATCM Third Grade Laboratory \\ of Chinese Medicine and Photonics \\ Technology, College of Biophotonics, \\ South China Normal University, \\ ${ }^{2}$ Guangdong Provincial Key Laboratory \\ of Sensor Technology and Biomedical \\ Instrument, School of Engineering, \\ Sun Yat-Sen University, Guangzhou, \\ Guangdong, People's Republic of \\ China; ${ }^{3}$ Faculty of Engineering, \\ University of Waterloo, Waterloo, \\ ON, Canada; ${ }^{4}$ Key Laboratory for \\ Polymeric Composite and Functional \\ Materials of Ministry of Education, \\ Sun Yat-Sen University, Guangzhou, \\ Guangdong, People's Republic of China
}

Correspondence: Jun Wu School of Engineering, Sun Yat-sen University, I 32 East Waihuan Road, Guangzhou Higher Education Mega Center, Guangzhou, Guangdong 510006, People's Republic of China Tel/fax +86 2039335046

Email wujun29@mail.sysu.edu.cn

Zhiming Liu

SATCM Third Grade Laboratory of Chinese Medicine and Photonics Technology, College of Biophotonics, South China Normal University, 55

Zhongshan Avenue West, Tianhe

District, Guangzhou, Guangdong 5 I063I,

People's Republic of China

Tel/fax+86208521 1920

Email liuzm021@।26.com
Abstract: The rapid development of near-infrared surface-enhanced Raman scattering (NIR SERS) imaging technology has attracted strong interest from scientists and clinicians due to its narrow spectral bandwidth, low background interference, and deep imaging depth. In this report, the graphene oxide (GO)-wrapped gold nanorods (GO@GNRs) were developed as a smart and robust nanoplatform for ultrafast NIR SERS bioimaging. The fabricated GO@ GNRs could efficiently load various NIR probes, and the in vitro evaluation indicated that the nanoplatform could exhibit a higher NIR SERS activity in comparison with traditional gold nanostructures. The GOs were prepared by directly pyrolyzing citric acid for greater convenience, andGO@GNRs were fabricated via a facile synthesis strategy. Higher NIR SERS activity, facile synthesis method, excellent biocompatibility, and superb stability make the GO@GNRs/probe complex promising nanoprobes for NIR SERS-based bioimaging applications.

Keywords: gold nanorods, graphene oxide, near-infrared bioimaging, surface-enhanced Raman scattering

\section{Introduction}

Surface-enhanced Raman spectroscopy (SERS) is a surface-sensitive technique that offers ultrasensitive fingerprint vibrational information, widely utilized in biosensing, biomolecular identification, drug delivery monitoring, and cancer theranostics. ${ }^{1-6}$ The SERS substrate can also be designed to serve as a sensitive Raman probe for bioimaging under in vitro and in vivo environments. ${ }^{7-11}$ Despite the remarkable progress in SERS, there are many bottlenecks hindering its development. The selection of metal, size, shape, and spacing of metal nanostructure have largely influenced the SERS effect. Shell-isolated nanoparticle-enhanced Raman spectroscopy (SHINERS) was developed to solve these limitations. ${ }^{12,13}$ This shell-isolated mode could offer higher detection sensitivity and diverse practical applications to various materials. Using graphene and its derivative as a novel isolating shell of Au nanostructure is proven to be very effective in bioimaging. ${ }^{13}$

Graphene is a single sheet of carbon atoms, known to exhibit superb Raman scattering properties due to its unique structure of electrons and phonons. ${ }^{14,15}$ As an important graphene derivative, graphene oxide (GO) has been widely used for a variety of biomedical applications. Zhang et al utilized GO as a platform to synthesize GO@ AuNPs or dye hybrids for fluorescence and SERS imaging of cells based on the optical properties of fluorescent dyes and characteristic fingerprints of GO, respectively, for Raman applications. ${ }^{16}$ In addition, GO can also be used as the coating material of 
metal nanostructures to fabricate core-shell nanocomposites, thereby allowing ultrasensitive SERS activity, better structural stability, as well as improved biocompatibility. ${ }^{17-20}$ GO-wrapped metal nanomaterials for SERS bioimaging by using the intrinsic Raman signals of GO (D and G bands) have also been developed in the recent years. ${ }^{21-23}$ However, this type of SERS probe with fixed pattern is limited for the multi-color Raman bioimaging. Gold nanorods (GNRs), a typical gold nanostructure, have received significant attention in the fields of material and life sciences due to their high extinction coefficient of longitudinal surface plasmon (LSPR) in the NIR region. ${ }^{19}$ Interestingly, the negative charge on the GO sheet surface enables utilization in the surface modification of positive-charged GNRs. ${ }^{24,25}$ Recently, GO-encapsulated GNRs have been applied to load anticancer agents for drug delivery, owing to the strong adsorption of aromatic molecules on graphene sheet. ${ }^{25,26}$ Thus, GO-wrapped GNRs are expected to perform as robust and applicable SERS substrates for dye-based SERS probe construction and biological imaging. However, previous studies concentrate intensively on the preparation and characterization of materials. As a consequence, application of SERS imaging has not been investigated deeply enough and was solely used for Raman enhancement of fluorescent dyes in a few studies. ${ }^{17,18}$

In this study, to expand the applications of GO-wrapped metal nanocomposites in bioimaging, GO-wrapped GNRs (GO@GNRs) were synthesized as smart nanoplatforms for ultrafast near-infrared (NIR) SERS bioimaging by a facile preparation method (Figure 1). The GO@GNR preparation process is applicable to a low-cost and a large-scale production. Moreover, GO@GNR-based NIR imaging systems have not been studied systematically. In comparison with SERS imaging alone, GO@GNR is able to exhibit excellent imaging effects and is non-toxic in nature. This work aimed to investigate the GO-wrapped GNRs as a general and accessible SERS bioimaging nanoplatform that can be extended to NIR fluorescent dye nanocarrier systems, thus providing enhanced NIR SERS images. This study currently provides a new application of SERS imaging.

\section{Materials and methods Synthesis of GNRs}

The GNRs were prepared in aqueous solution by using a seedless method adapted from the initial research work of El-Sayed et al. ${ }^{27}$ An initial solution was first prepared with five components: $25 \mathrm{~mL}$ of cetyltrimethylammonium bromide (CTAB, $0.2 \mathrm{M}$ ) was added into a $\mathrm{HAuCl}_{4}$ aqueous solution (25 mL, $1 \mathrm{mM}$ ) while gently shaking at room temperature. $\mathrm{AgNO}_{3}(4 \mathrm{mM})$ and $\mathrm{HCl}(50 \mu \mathrm{L}, 37 \%)$ were then added while shaking gently to obtain a $\mathrm{pH}$ of $1-1.15$. The amount of $\mathrm{AgNO}_{3}$ could be adjusted to prepare different GNRs. Consequentially, ascorbic acid $(350 \mu \mathrm{L}, 78.8 \mathrm{mM})$ was added, and the initially red solution $\left(\mathrm{HAuCl}_{4}, \mathrm{CTAB}\right)$ became uncolored. In the second stage, a tiny volume $(75 \mu \mathrm{L})$ of ice-cold $\mathrm{NaBH}_{4}(0.01 \mathrm{M})$ was injected to the unstirred growth solution and left to react overnight at $28^{\circ} \mathrm{C}$, and the resulting final mixture was transferred to a waterbath. The final product was purified by centrifugation ( $10,000 \mathrm{rpm}, 30$ minutes) three times and then resuspended in $25 \mathrm{~mL}$ deionized water.

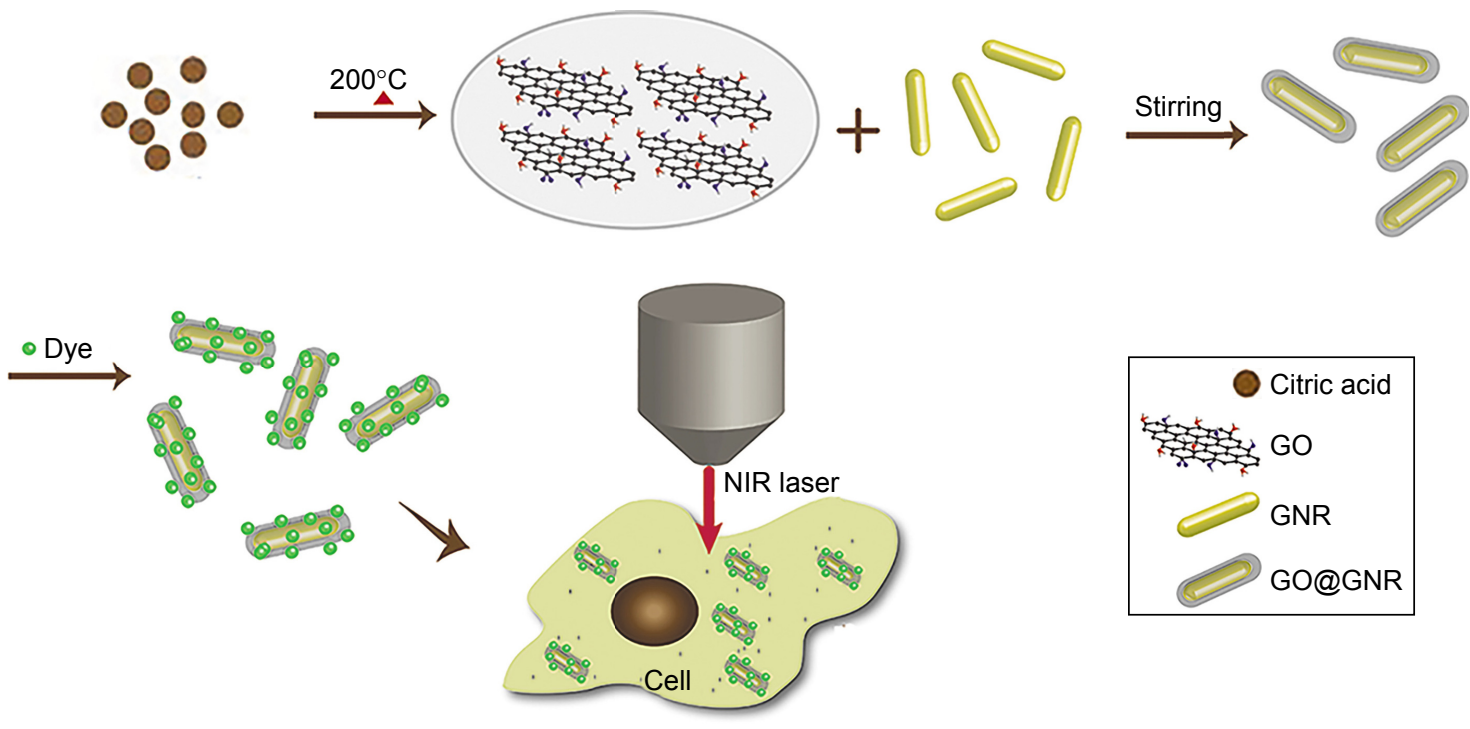

Figure I Synthesis of GO@GNRs as NIR SERS nanoprobes.

Abbreviations: GO, graphene oxide; GNR, gold nanorod; GO@GNRs, GO-wrapped GNRs; NIR, near-infrared; NIR SERS, near-infrared surface-enhanced Raman scattering. 


\section{Synthesis of graphene oxide}

GO was prepared by directly pyrolyzing citric acid (CA). ${ }^{28}$

$2 \mathrm{~g}$ CA was added to a $5 \mathrm{~mL}$ beaker and heated to $200^{\circ} \mathrm{C}$ by using a heating mantle. After a time period of 5 minutes, the CA was liquated. Subsequently, the color of the liquid changed from colorless to pale yellow, to orange, and finally transformed into a black solid during the period of about 2 hours. The black solid was dissolved with $50 \mathrm{~mL}$ of $10 \mathrm{mg} / \mathrm{mL} \mathrm{NaOH}$ solution and further neutralized with the same concentration of $\mathrm{NaOH}$, resulting in the production of an aqueous solution of GO.

\section{Fabrication of GO@GNRs}

GNRs could be bonded on the surface of GO via electrostatic interaction. Typically, the GNR solution is diluted to a concentration of OD value 1.0. Consequentially, $10 \mathrm{~mL}$ GNR dispersion was added to the GO solution with continuous stirring at room temperature. After stirring for 6 hours, the solution was then centrifuged (10,000 rpm, 30 minutes) twice to remove excess free GO nanosheets. Precipitates were in turn collected and redispersed in $1 \mathrm{~mL}$ of deionized water.

\section{Assembly of SERS probes}

The SERS activities of GO@AuNR at the NIR range can be investigated by using the different dye molecules as the probes. Four commonly used dye molecules (IR-780, IR-820, MB, and 2-ATP) were added into GO@GNR solution with 1.0 OD value, respectively, followed by stirring for 3 hours. Consequentially, $1 \mathrm{mg} / \mathrm{mL}$ PSS was added to the mixture. After stirring for 12 hours, the solution was centrifuged $(10,000 \mathrm{rpm}$, 30 minutes) twice, and the precipitates were collected.

\section{Characterization of materials}

UV-Vis absorption spectra of the nanostructures were recorded by using a UV-Vis spectrometer (UV-3200S; MAPADA, Shanghai, People's Republic of China). Morphological features of the GNRs and GO@GNRs were characterized by Philips TECNAI 10 transmission electron microscopy (TEM). Atomic force microscopy (AFM) images were taken in tapping mode with the SPM Dimension 3100 from Veeco.

\section{SERS experiments}

Raman spectra were recorded by using a Renishaw inVia microspectrometer (Derbyshire, UK) with excitation wavelengths of 514.5 and $785 \mathrm{~nm}$ generated by using an $\mathrm{Ar}^{+}$laser and a semiconductor laser, respectively. The SERS samples were obtained by adding $10 \mu \mathrm{L}$ GO@GNR solution $(1 \mathrm{mM})$ to the same volume of SERS dyes. GNRs with similar gold concentrations were also prepared as the control. A $20 \times$ objective lens ( $\sim 3 \mu \mathrm{m}$ laser spot size $)$ was used to focus the laser beam and to collect the Raman signal.

\section{In vitro NIR SERS imaging experiments}

Mouse breast adenocarcinoma (4T1) cells, adenocarcinomic human alveolar basal epithelial (A549) cells, human epidermoid carcinoma (KB) cells, human tongue carcinoma (CAL-27) cells, and human osteosarcoma (143B) cells were all purchased from the American Type Culture Collection (ATCC, Manassas, VA, USA). They were cultivated in Dulbecco's Modified Eagle's Medium (DMEM) or Roswell Park Memorial Institute-1640 medium supplemented with $10 \%$ fetal bovine serum and antibiotics in a humidified atmosphere with $5 \% \mathrm{CO}_{2}$ at $37^{\circ} \mathrm{C}$. The cells were first seeded onto sterile quartz substrates in cell culture Petri dishes and incubated at $37^{\circ} \mathrm{C}$. After the cell attachment, the growth medium was replaced with a fresh medium containing SERS probes at a concentration of $100 \mu \mathrm{M}$. Cells were incubated for another 6 hours. Subsequently, the medium was removed and the remaining contents were washed with phosphate-buffered saline five times. These cells were then visualized by using a Renishaw inVia Raman microscope system with a $785 \mathrm{~nm}$ laser excitation (streamline mode at wavenumber center $1,200 \mathrm{~cm}^{-1}$ ).

\section{Cytotoxicity assessments}

The ideal SERS probe must have low toxicity or be completely non-toxic in nature with constitutional stability under storage. The cytotoxicity of the related gold nanostructures was evaluated by the classic methyl thiazolyl tetrazolium (MTT) viability assay. A549 cells were cultured in DMEM containing 10\% fetal bovine serum and incubated for 24 hours with GNRs and Au@GNRs with various concentrations ranging from 0 to $200 \mu \mathrm{M}$, respectively. WST-1 reagent (Roche, Mannheim, Germany) was then added into the medium $(1: 10, \mathrm{v} / \mathrm{v})$. After a period of 1 hour, the absorbance intensities of each sample at $440 \mathrm{~nm}$ were measured by using an automatic microplate reader.

\section{Photothermal effects of NIR SERS probes} KB cells were incubated with GO@GNRs/IR-780 $(100 \mu \mathrm{M})$ for 6 hours, and the samples were scanned to collect the Raman signal and Raman images. This operation was carried out by a Renishaw inVia confocal Raman system (coupled to a Leica DM-2500M microscope). Consequentially, the cells were irradiated with a NIR laser $(785 \mathrm{~nm}, 0.1 \mathrm{~W})$ twice every 2 minutes and Raman imaged after each laser irradiation. All preventive measures were carried out during the laser experiments. 


\section{Results and discussion}

Synthesis and characterization of GO@GNRs

In this experiment, fabrication of GO@GNRs was attempted by directly adding CTAB-capped GNR dispersion to the bare GO dispersion with vigorous stirring due to the electrostatic effect between the negatively charged GO sheets and the positively charged GNRs. GO was prepared by directly pyrolyzing CA. The AFM image in Figure 2A shows further details about the GO structure. The results from the AFM image indicated that GO consists of mostly sheets with sizes ranging from tens to hundreds nanometers (average $100 \mathrm{~nm}$ ).

\section{A}
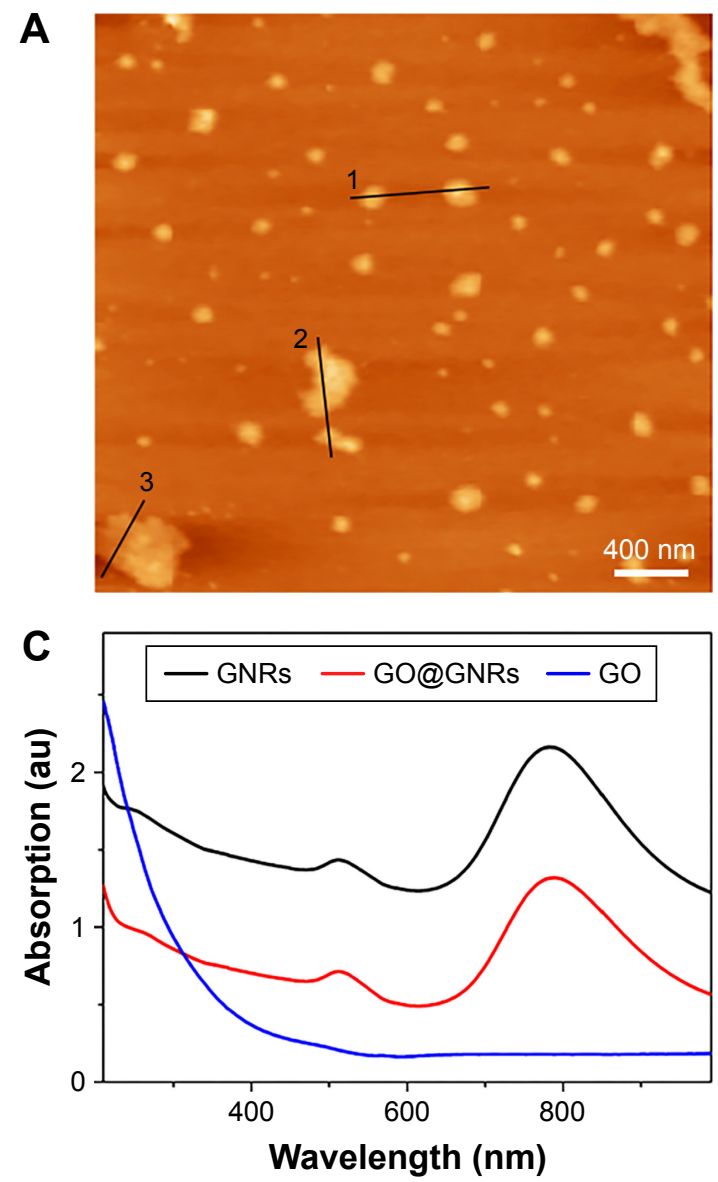

E

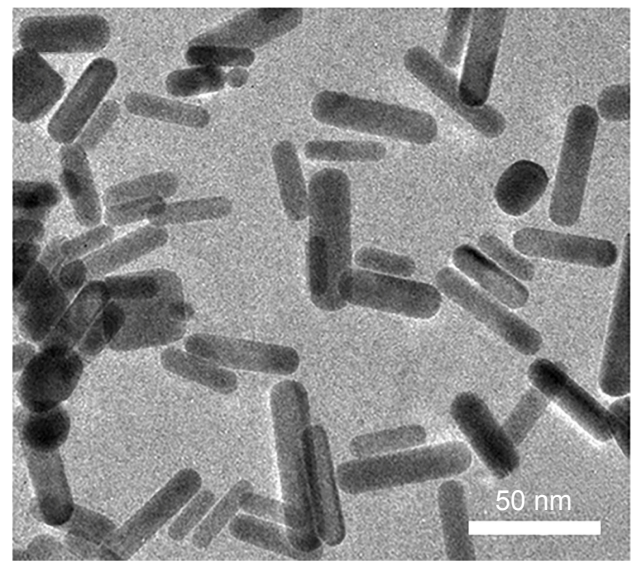

B

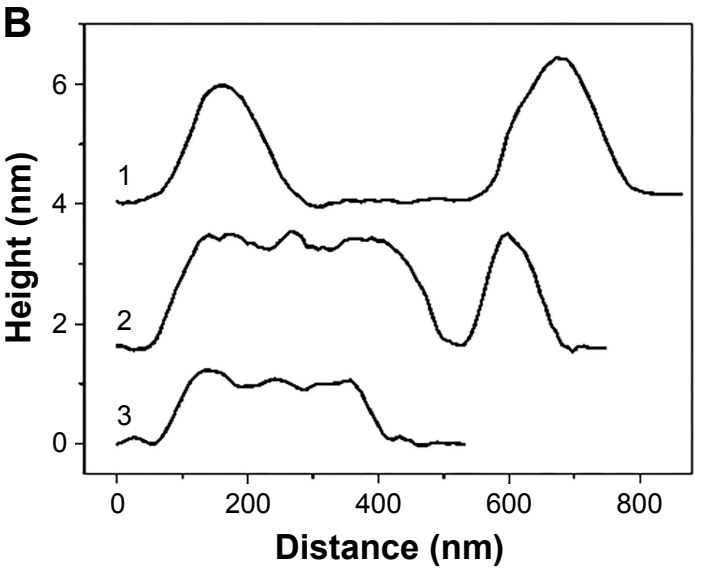

D

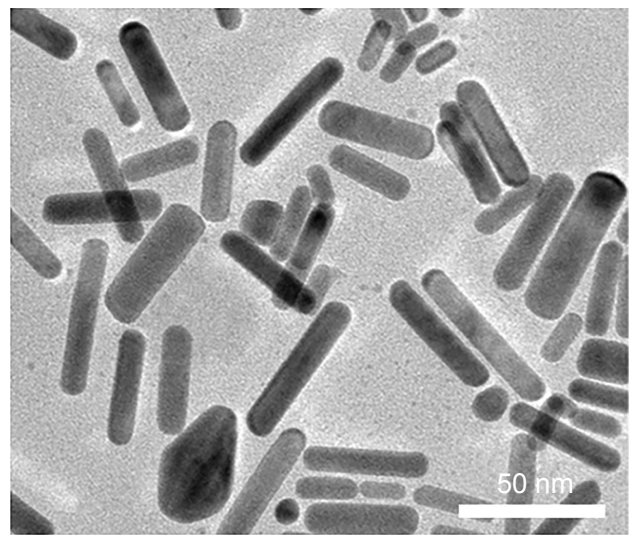

$\mathbf{F}$

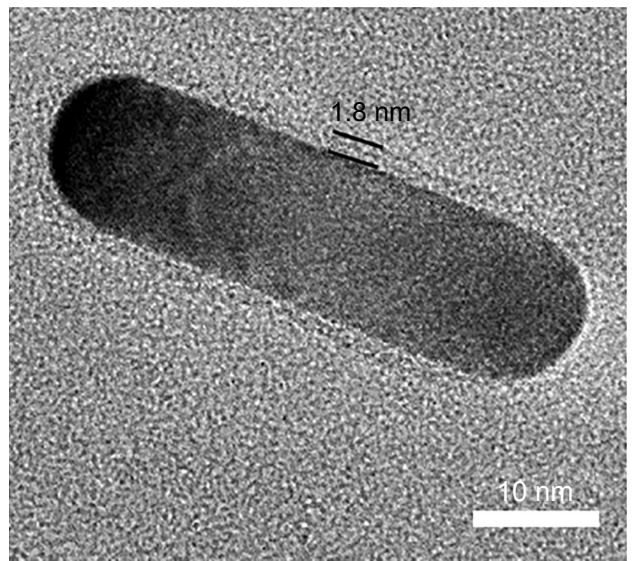

Figure 2 Characterization of the nanomaterials.

Notes: (A) AFM image of GO nanosheets adsorbed on a mica substrate. Black lines (I-3) indicate the stacking of GO layers. (B) The height profiles of GO marked (I-3) in the AFM image. (C) UV-Vis-NIR absorption spectra of GO, GNRs, and GO@GNRs. (D) TEM image of GNRs. (E) TEM image of GO@GNRs. (F) TEM image of GO@ GNRs with higher magnification.

Abbreviations: AFM, atomic force microscopy; GO, graphene oxide; GNR, gold nanorod; GO@GNRs, GO-wrapped GNRs; NIR, near infrared; TEM, transmission electron microscopy. 
Figure $2 \mathrm{~B}$ shows the heights of the obtained GO appeared in three areas in Figure 2A, and the results show that the thickness of GO is $\sim 1.8 \mathrm{~nm}$, which indicates the formation of single- or two-layered GO.

The binding of GO with GNRs is confirmed by UV-VisNIR spectroscopy (Figure 2C). CTAB-stabilized GNRs contain two special peaks including a strong LSPR in the long-wavelength region $(\sim 780 \mathrm{~nm})$ and a weak transverse surface plasmon oscillation in the short-wavelength region $(\sim 515 \mathrm{~nm})$. However, the optical absorption peak of GO in NIR region could not be observed even though it displayed a broad UV-Vis absorption. After incorporation with GO, the LSPR peak of GO@GNRs displayed a red shift plasmon band in comparison with that of pure GNRs, which could mainly be ascribed to the change of the coefficient of light extinction in the microenvironment of the nanostructures.

The morphology of GNRs and GO@GNR was investigated by TEM (Figure 2D and E). As shown in Figure 2E, the prepared GO@GNRs are quite uniformly rod shaped. The thickness of the GO shell was approximately $1.8 \mathrm{~nm}$, as measured from the TEM image with higher magnification (Figure 2F).

\section{The NIR SERS activities of nanoprobes}

The importance of an efficient wrapping of GO around GNRs in order to obtain a good SERS effect is shown in Figure 3. Six dyes were used as probe molecules to investigate the SERS activities. The black lines (1) show the normal Raman spectra of dye molecules and the free dye molecules represent no Raman signal. The green lines (3) represent the GO@ GNRs irradiated with a visible laser $(514.5 \mathrm{~nm})$, where no obvious Raman enhancements are observed. On contrary, by solely using the MG and $\mathrm{CV}$ as the probes, slight Raman enhancements could be detected due to the fact that their absorption peaks are closer to the laser peak (514.5 $\mathrm{nm})$.

The red (2) and blue (4) lines represent the GNRs and GO@GNRs under NIR laser excitation (785 nm), respectively. Both GNRs and GO@GNRs exhibited intense SERS signals. However, in comparison with GNRs, the GO@ GNRs possessed a much stronger Raman signal-enhancing ability. In these spectra, the increase in signal enhancement from GO@GNRs to GNRs is 9.6-fold when using the IR-780 as the dye probe and 14.5-fold when using the IR-820. These enhancements are 13.8- and 12.0-fold for MB-based nanoprobes and MG-based nanoprobes, as well as 4.5- and 4.7-fold for CV-based nanoprobes and 2-ATP-based nanoprobes. Overall, these results imply that wrapping of GO around the GNRs shows remarkable NIR activity that can significantly improve the NIR SERS effect of Raman reporters.

\section{Optimize the condition of NIR SERS}

With the discovery of prominent and permanent SERS features, the capabilities of IR-780 as dye probe of NIR SERS were investigated. Figure 3 shows that the absorption peak of IR-780 is $\sim 780 \mathrm{~nm}$ and hence can match the excitation wavelength to exhibit a SERRS (surface-enhanced resonance Raman scattering) effect, thus improving the enhancement of SERS effect. GNRs with different LSPR peaks (680, 700, 760, 780, and $810 \mathrm{~nm}$ ) were prepared similar to GO@ GNRs (Figure 4A). As shown in Figure 4B, the GO@GNRs with LSPR peak at $780 \mathrm{~nm}$ exhibit extremely intense SERS signals and highly enhanced activities of NIR SERS. These results are mainly due to similarity between LSPR peaks of nanostructures and the excitation light absorption peak, which can produce additional enhancements of NIR SERS.

To investigate the range of concentration of dye molecules that can exhibit SERS effect, the GO@GNRs with different concentrations ( $5 \mathrm{nM}, 50 \mathrm{nM}, 0.5 \mu \mathrm{M}, 5 \mu \mathrm{M}$, and $50 \mu \mathrm{M}$ ) of dye molecules were prepared. The results indicated that the SERS effect of dyes is concentration dependent. As shown in Figure $5 \mathrm{~A}$, when the concentration decreased to $5 \mathrm{nM}$, the SERS signals can still be detected. However, the spectral intensity greatly increases when the dye concentration increases. In order to investigate the stability of the dye molecule, the GO@GNRs were detected with IR-780 for 1 hour. The results reveal that the intensity of this peak for GO@GNRs solutions remains constant over the 1-hour period (Figure 5B), thus showing that the particles are stable for SERS.

\section{In vitro cytotoxicity}

The cytotoxicity of GO@GNRs was examined in A549 cells (Figure 6). It was noted that the cytotoxicity of GNRs is concentration dependent. On increasing the GNR concentration to $200 \mu \mathrm{M}$, the cell viability decreased to $29.1 \%$. In comparison with GNRs, the GO@GNRs exhibited a greatly enhanced biocompatibility. Moreover, increasing the GO@GNR concentration to $200 \mu \mathrm{M}$, it was seen that $90 \%$ of the A549 cells were still alive. It is well known that the cytotoxicity induced by GNRs is highly dependent on the amount of residual CTAB on the surface of GNRs. ${ }^{29}$ The encapsulation of GNRs inside GO shell enables lesser contact with the surrounding environments, resulting in the enhanced biocompatibility of GO@GNRs.

\section{Ultrafast NIR SERS bioimaging based on GO@GNRs}

In comparison with the control group (Figure 7A-C), cells incubated with GO@GNR/IR-780 (Figure 7E-G) exhibit distinguishable and heterogeneous Raman signals, mainly 

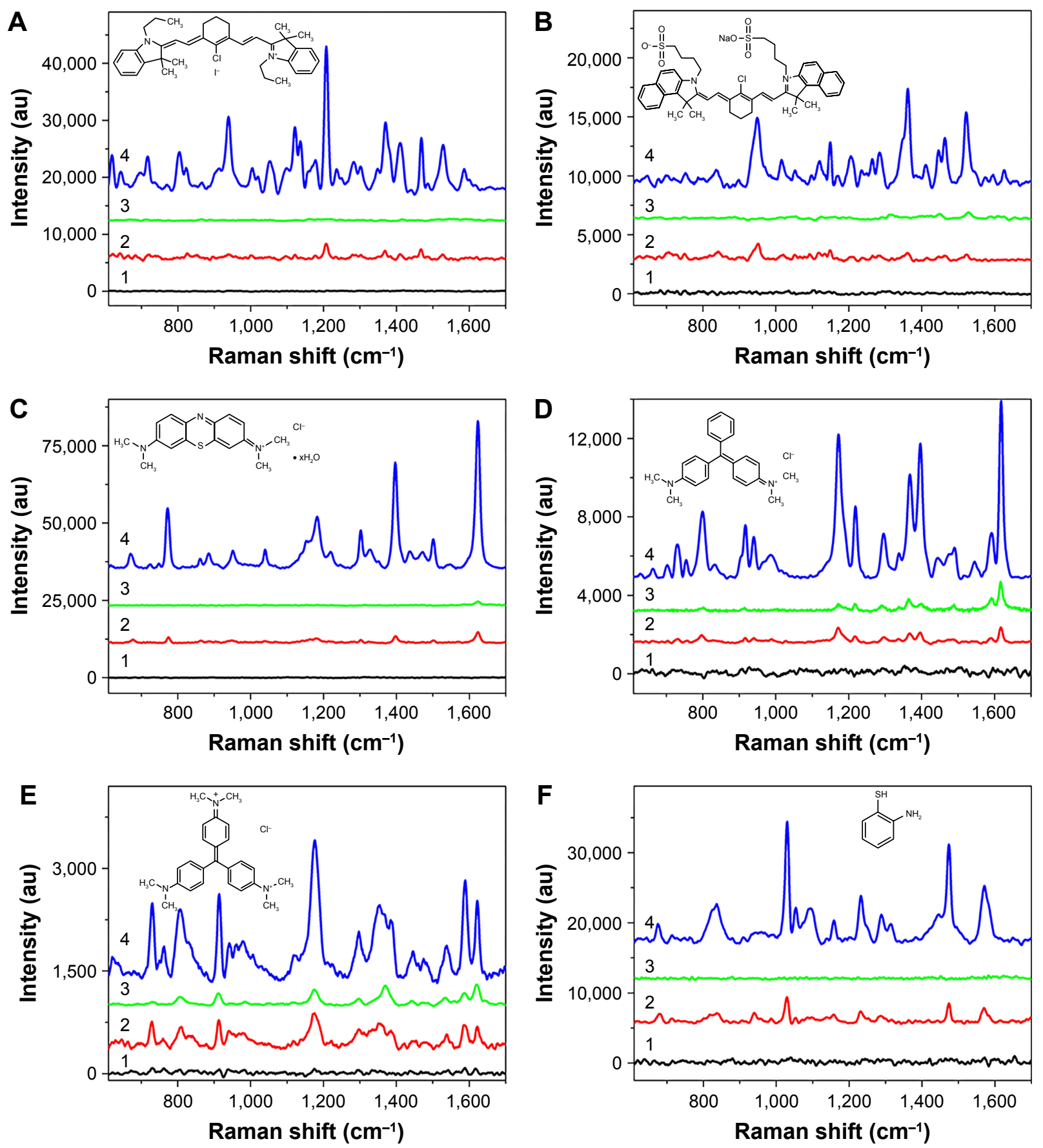

Figure 3 The SERS activities of GO@GNRs using (A) IR-780, (B) IR-820, (C) MB, (D) MG, (E) CV, or (F) 2-ATP as the Raman reporter.

Notes: Spectral lines 1, 2, 3, and 4 represent the normal Raman spectrum of dye, SERS spectrum induced by GNRs under $785 \mathrm{~nm}$ laser irradiation, SERS spectra induced by GO@GNRs under lasers centered at 514.5 and 785 nm, respectively. The insets show the molecular structures of the Raman reporters.

Abbreviations: SERS, surface-enhanced Raman scattering; GO, graphene oxide; GNR, gold nanorod; GO@GNRs, GO-wrapped GNRs.

distributed in the cytoplasmic area. The SERS images when closely overlaid with the bright-field images (Figure 7H-J) revealed the availability of GO@GNR/IR-780 as novel NIR SERS labels for cellular SERS imaging in 143B cells. As shown in Figure 7D, the SERS signals of IR-780 can be observed clearly in the spectral lines collected from the cells containing SERS probes, while no signals are detected in the control group. The Raman spectra (three gray lines) obtained from different cytoplasmic compartments show the inconsistency of Raman intensity, thus indicating heterogeneous SERS probe distribution. Furthermore, the imaging of NIR SERS probes is significantly faster than other Raman probes, ${ }^{30-32}$ thus requiring a very short integration time $(\sim 0.04$ seconds per pixel). Multiple enhancement mechanisms are proposed to explain the ultrafast NIR SERS imaging induced by GO@GNR/IR-780 probe: 1) SERRS effect occurs between 

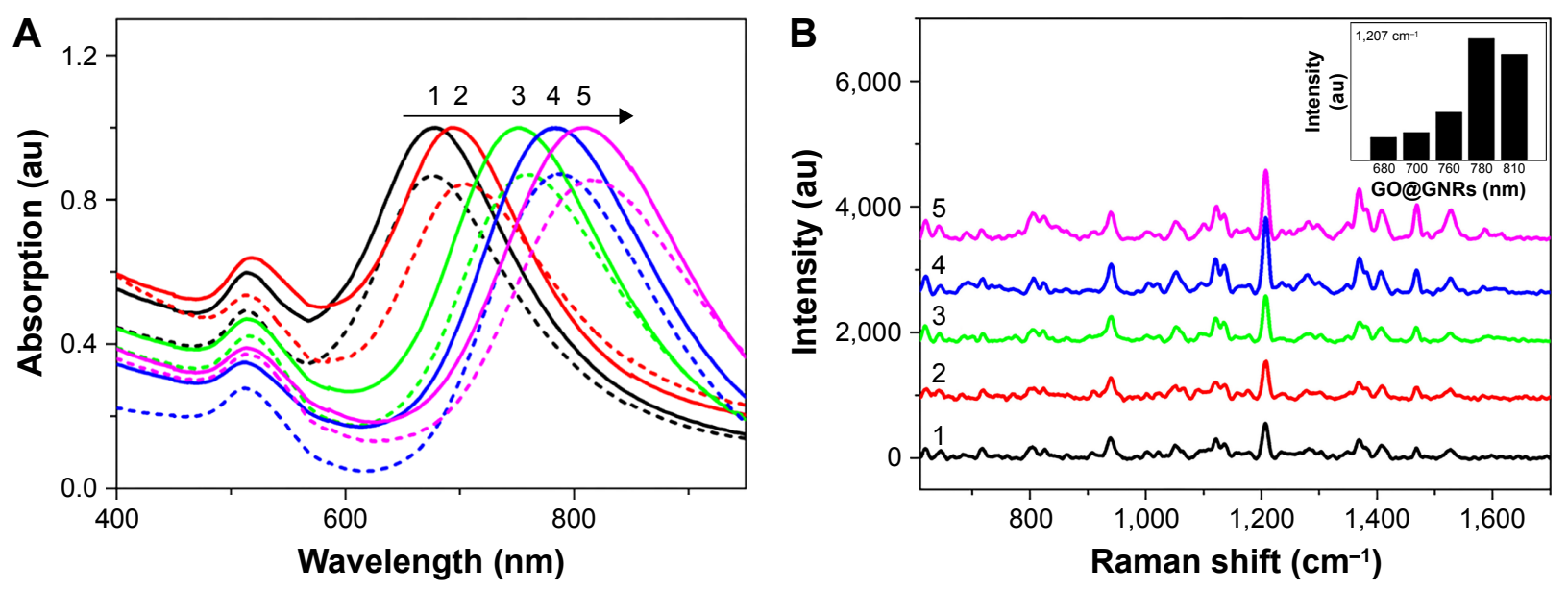

Figure 4 Optimization of the NIR SERS activities of GO@GNRs.

Notes: (A) UV-Vis absorption spectra of GNRs (solid lines) and GO@GNRs (dotted lines) with different aspect ratios. (B) Raman spectra obtained from the GO@GNRs with different LSPR peaks (680 nm [1], $700 \mathrm{~nm}$ [2], $760 \mathrm{~nm}$ [3], $780 \mathrm{~nm}$ [4], and $810 \mathrm{~nm}$ [5]). Inset shows the SERS intensities of I,207 cm ${ }^{-1}$.

Abbreviations: GO, graphene oxide; GNR, gold nanorod; GO@GNRs, GO-wrapped GNRs; LSPR, longitudinal surface plasmon; NIR near-infrared; SERS, surface-enhanced Raman scattering.

the NIR dye and the matched excitation wavelength; ${ }^{33}$ the LSPR peak of GNRs may also contribute to the improved SERS signals of the NIR dye. ${ }^{34}$ 2) The charge transfer between graphene sheet and the Raman reporter appears under laser irradiation, resulting in graphene-enhanced Raman scattering (GERS) effect. ${ }^{35} 3$ ) The enrichment of dye molecules on the GO sheet may also cause intensive SERS signals, eventually leading to ultrafast NIR SERS imaging. ${ }^{36}$

To explore whether SERS probes are applicable to different cells, the NIR SERS images were acquired from 4T1 cells, A549 cells, KB cells, and CAL-27 cells. The results denoted that all cells exhibit excellent imaging effects like 143B cells (Figure 8A-H). No significant difference is observed in the Raman spectral pattern between the cells and IR-780 (Figure 8I), confirming that NIR SERS probes GO@GNR/IR-780 are also available in these cells.
In comparison with the imaging effects of SERS probes containing different Raman active molecules, the NIR SERS images were acquired from cells by using different dye-based SERS probes. Accurate and clear NIR SERS images are observed with all dyes (Figure 9A-L). SERS spectral lines attributed to Raman reporter molecules are kept separate from the cell lines (Figure 9M). The resulting data validate the versatility of GO@GNR-based NIR SERS probes and uncover the potential of GO@GNRs for multi-color Raman bioimaging.

\section{Photothermal effects and real-time monitoring of NIR SERS probes}

As shown in the UV-Vis-NIR absorbance spectra (Figure 10A), the NIR SERS probe exhibits the dramatic NIR optical absorbance, indicating a promising potential for photothermal
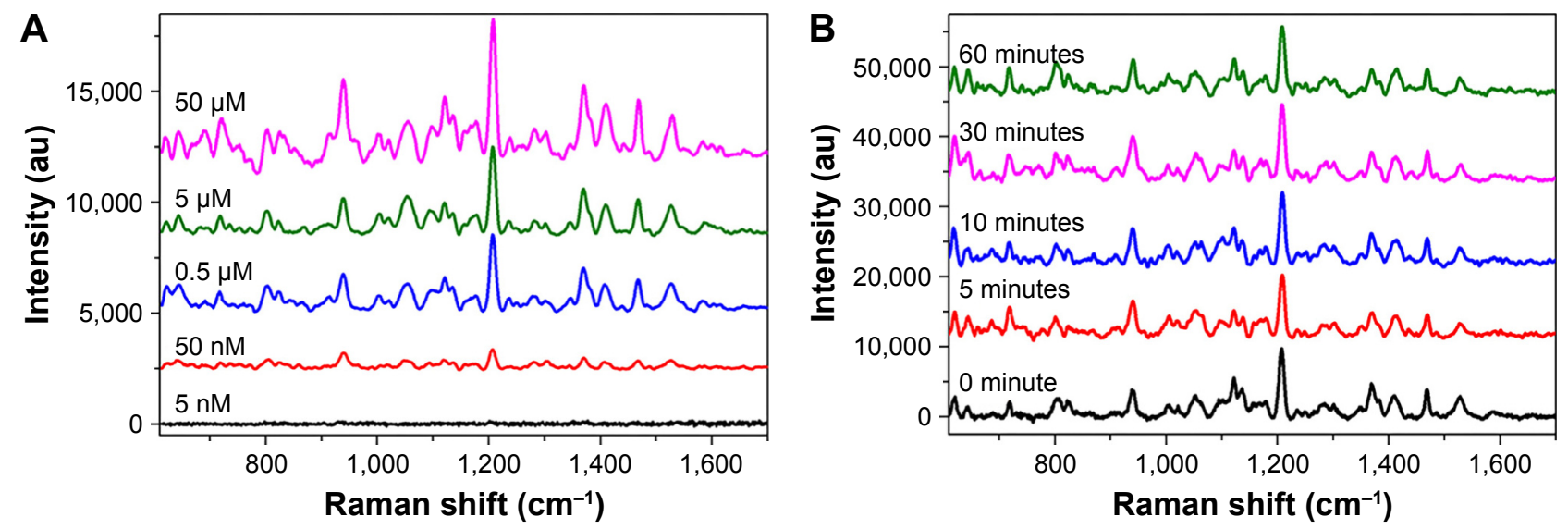

Figure 5 (A) Concentration-dependent SERS effect of IR-780 induced by GO@GNRs. (B) The SERS stability of the GO@GNR-based nanoprobe. Abbreviations: GO, graphene oxide; GNR, gold nanorod; GO@GNRs, GO-wrapped GNRs; SERS, surface-enhanced Raman scattering. 


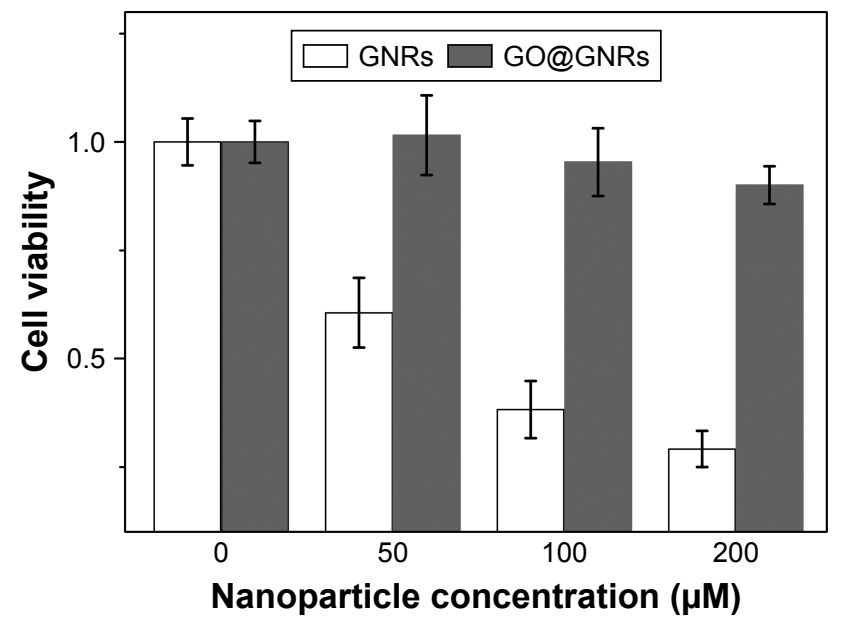

Figure 6 In vitro cytotoxicity of GNRs and GO@GNRs against A549 cells. Abbreviations: GO, graphene oxide; GNR, gold nanorod; GO@GNRs, GOwrapped GNRs.

cancer therapy. The kill efficacies of GO@GNR/IR-780induced photothermal cell ablation were then investigated. After exposure to the NIR laser, the originally well-defined cell structure collapsed. This was observed in real time while monitoring KB cells containing SERS probes by bright-field images (Figure 10B, first column) showing that the cell bulk shrinkage was due to laser ablation, accompanied by irreversible membrane blebbing (indicated by the arrows). Cells suffered greater photoactivated damage after the second irradiation treatment, resulting in the volume of cells being smaller than the cells in the previous treatment. As a result, it is unable to observe the internal structure of cells. Besides, the increase in the amount of cavitation bubbles and structural extension is a generally accepted sign of cell death.

The physiological phenomenon shown in the bright-field images is accurately reflected by using the cell SERS imaging (Figure 10B, second column). Before the laser irradiation, SERS signals are detected in the cytoplasm, indicating that SERS probes cannot enter into the nucleus. After the laser irradiation, the distribution area of SERS signals significantly reduced. For example, cell 1 and cell 2 (Figure 10B, third column, labels 1 and 2) display an intense and homogeneous signal distribution before exposure to laser. Contrarily, both smaller SERS imaging area and attenuated signal partially are observed after laser irradiation, revealing that cellular melting has occurred, which can be also demonstrated from
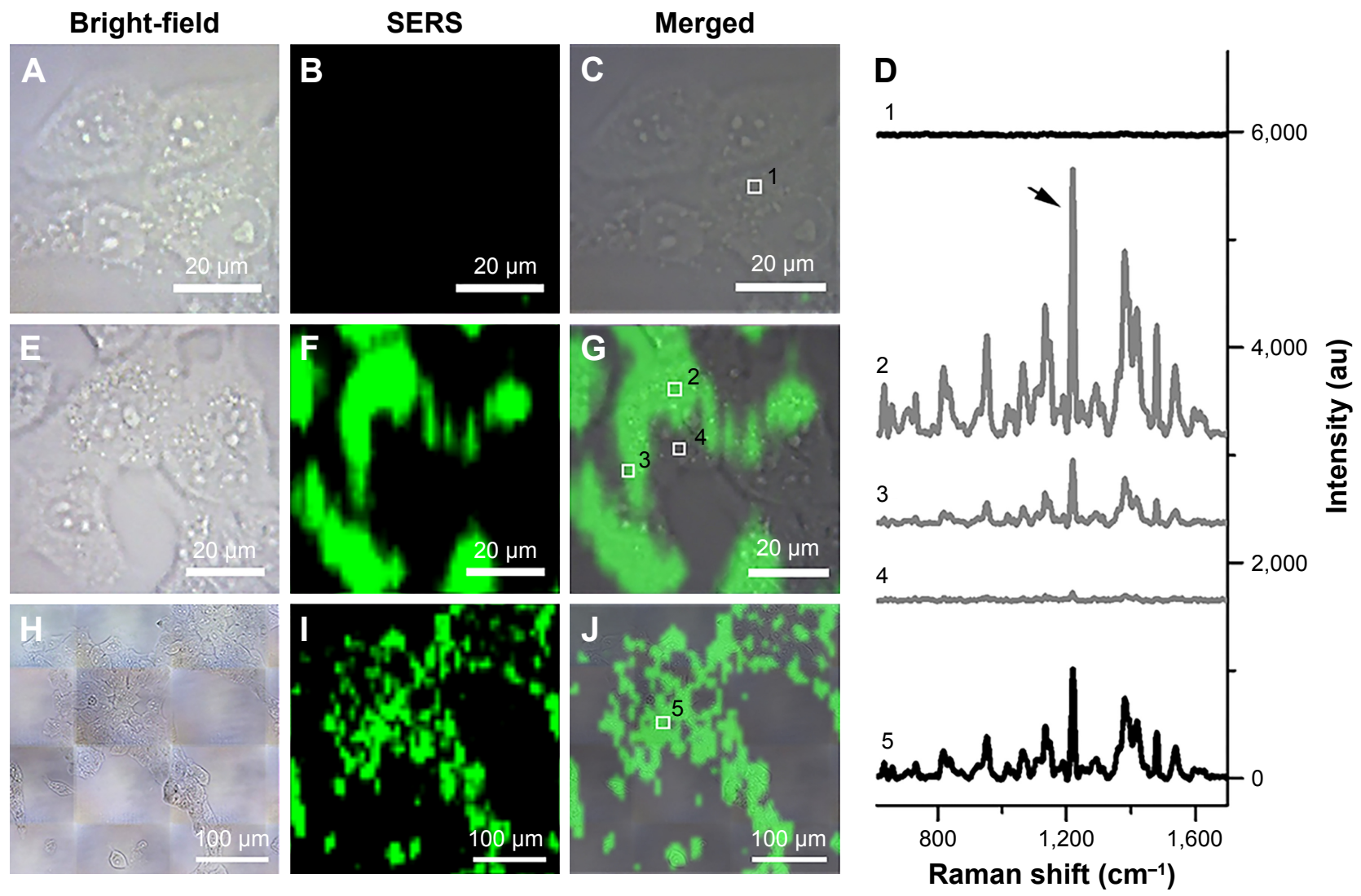

Figure 7 Ultrafast NIR SERS bioimaging of I43B cells using GO@GNR/IR-780 as the SERS probes.

Notes: Cells incubated in medium without GO@GNR/IR-780 (A-C) are shown as control. The images (E-G) and large-scale images $(\mathbf{H}-\mathbf{J})$ are acquired after incubation with GO@GNR/IR-780 for 6 hours. (D) The Raman spectral lines of the different spots marked in the cytoplassmic compartments (white boxes marked as I-5 in C, $\mathbf{G}$, and $\mathbf{J})$. The arrow indicates the Raman peaks used for SERS imaging.

Abbreviations: GO, graphene oxide; GNR, gold nanorod; GO@GNRs, GO-wrapped GNRs; NIR, near-infrared; SERS, surface-enhanced Raman scattering. 

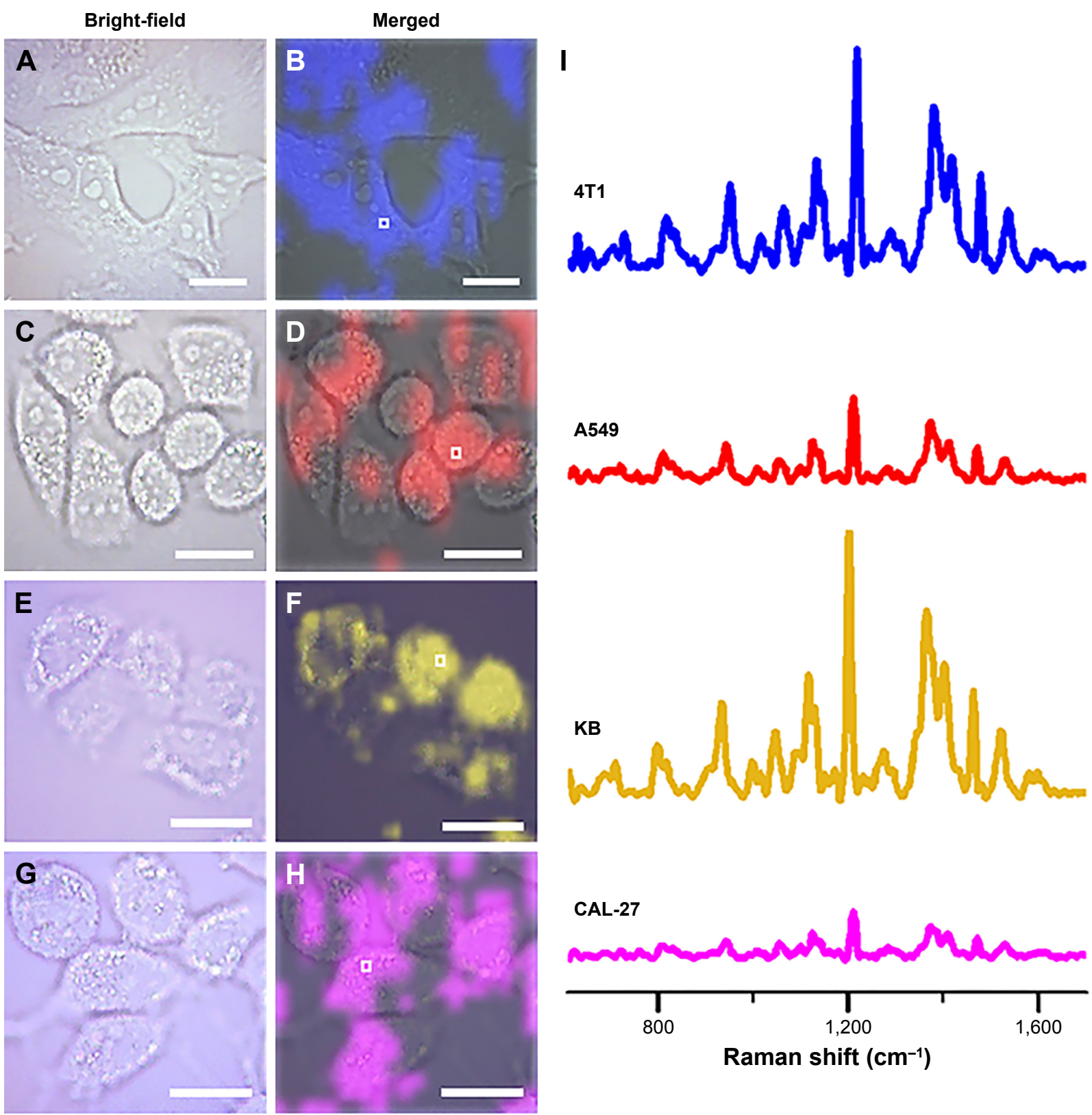

Figure 8 NIR SERS bioimaging of different cells using GO@GNR/IR-780 as SERS probes.

Notes: Typical bright-field images (A, C, E, G) and merged images (B, D, F, H) of 4TI cells, A549 cells, KB cells, and CAL-27 cells. Image I shows the Raman spectra extracted from the intracellular regions (white boxes marked in $\mathbf{B}, \mathbf{D}, \mathbf{F}$ and $\mathbf{H}$ ) of the different cancer cells. Scale bars: $20 \mu \mathrm{m}$.

Abbreviations: GO, graphene oxide; GNR, gold nanorod; GO@GNRs, GO-wrapped GNRs; NIR, near-infrared; SERS, surface-enhanced Raman scattering.

the reduction of cellular average SERS signals (Figure 10C and D). The obvious and continuous decrease in the SERS intensity is seen in the spectral line of NIR SERS probes incubated with $\mathrm{KB}$ cells treated with or without the laser. Furthermore, a significant SERS signal is discovered in the original low or undetected signal area (Figure 10B, third column, asterisk area) followed by the destruction of integrated cell structure. This result shows that laser irradiation would lead to an influx of cellular contents. As shown in Figure 10E, the relative SERS intensity increased dramatically with laser irradiation, demonstrating that migration of cell inclusions would occur due to cell internal structure change and damage induced by laser ablation. ${ }^{37}$

\section{Conclusion}

In summary, NIR SERS imaging is a promising molecular imaging technology currently due to its narrow spectrum feature, low background interference, and depth of imaging performance. GNR cores and GO shell nanocomposites (GO@GNRs) were successfully synthesized through a facile mixing method. The preparation process of GO@GNRs achieves low-cost and large-scale production. In comparison 


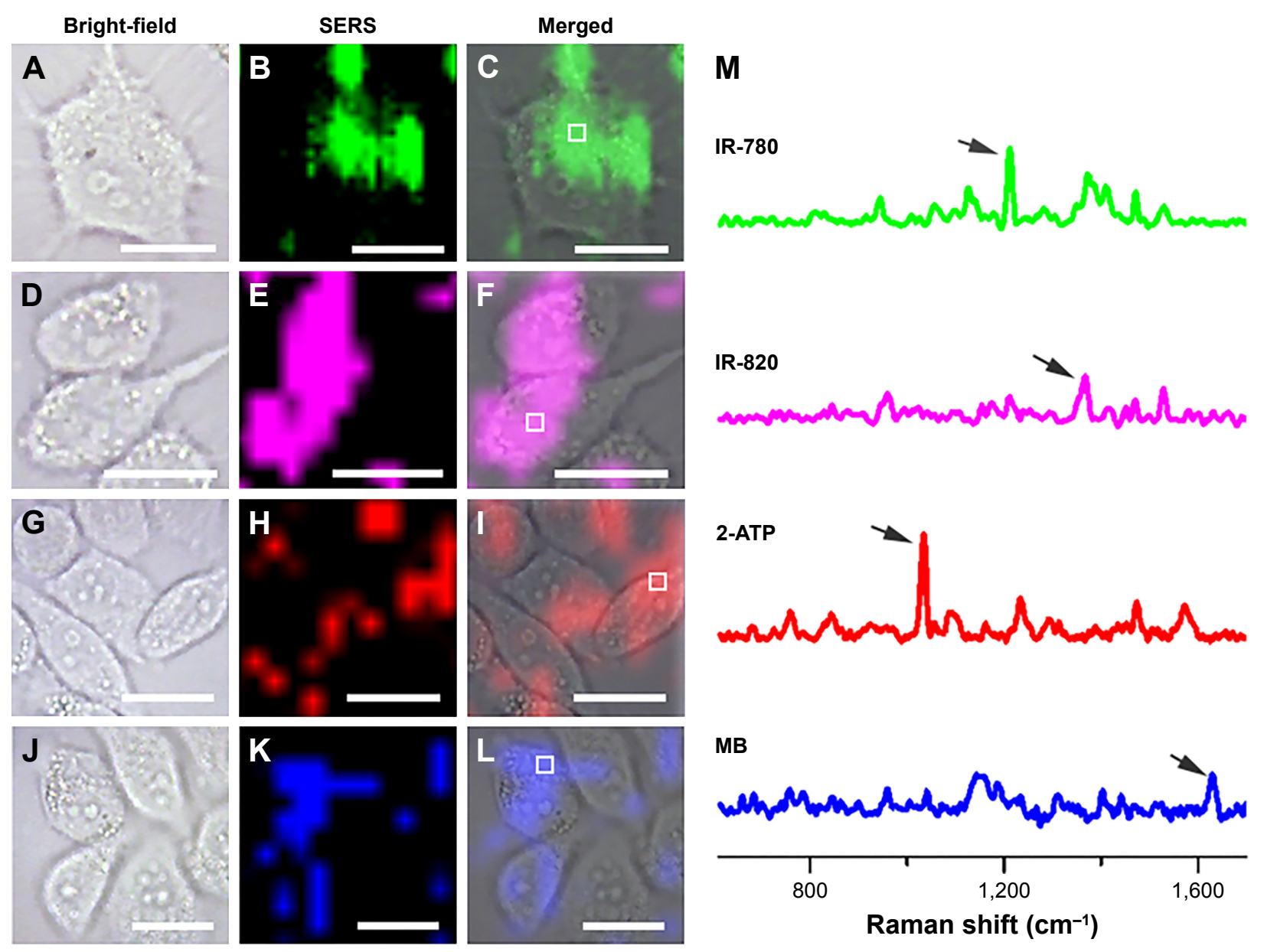

Figure 9 NIR SERS bioimaging of A549 cells using GO@GNR-loaded different Raman active reporter molecules as SERS probes.

Notes: The images are acquired after incubation with GO@GNR/IR-780 (A-C), GO@GNR/IR-820 (D-F), GO@GNR/2-ATP (G-I), and GO@GNR/MB (J-L) for6 hours. Image $\mathbf{M}$ Shows the Raman spectra extracted from the intracellular regions (white boxes marked in $\mathbf{C}, \mathbf{F}, \mathbf{I}$, and $\mathbf{L}$ ) of A549 cells incubated with the different SERS probes. The arrows indicate the Raman peaks used for SERS imaging of four different molecules. Scale bars: $20 \mu \mathrm{m}$.

Abbreviations: GO, graphene oxide; GNR, gold nanorod; GO@GNRs, GO-wrapped GNRs; NIR, near-infrared; SERS, surface-enhanced Raman scattering.
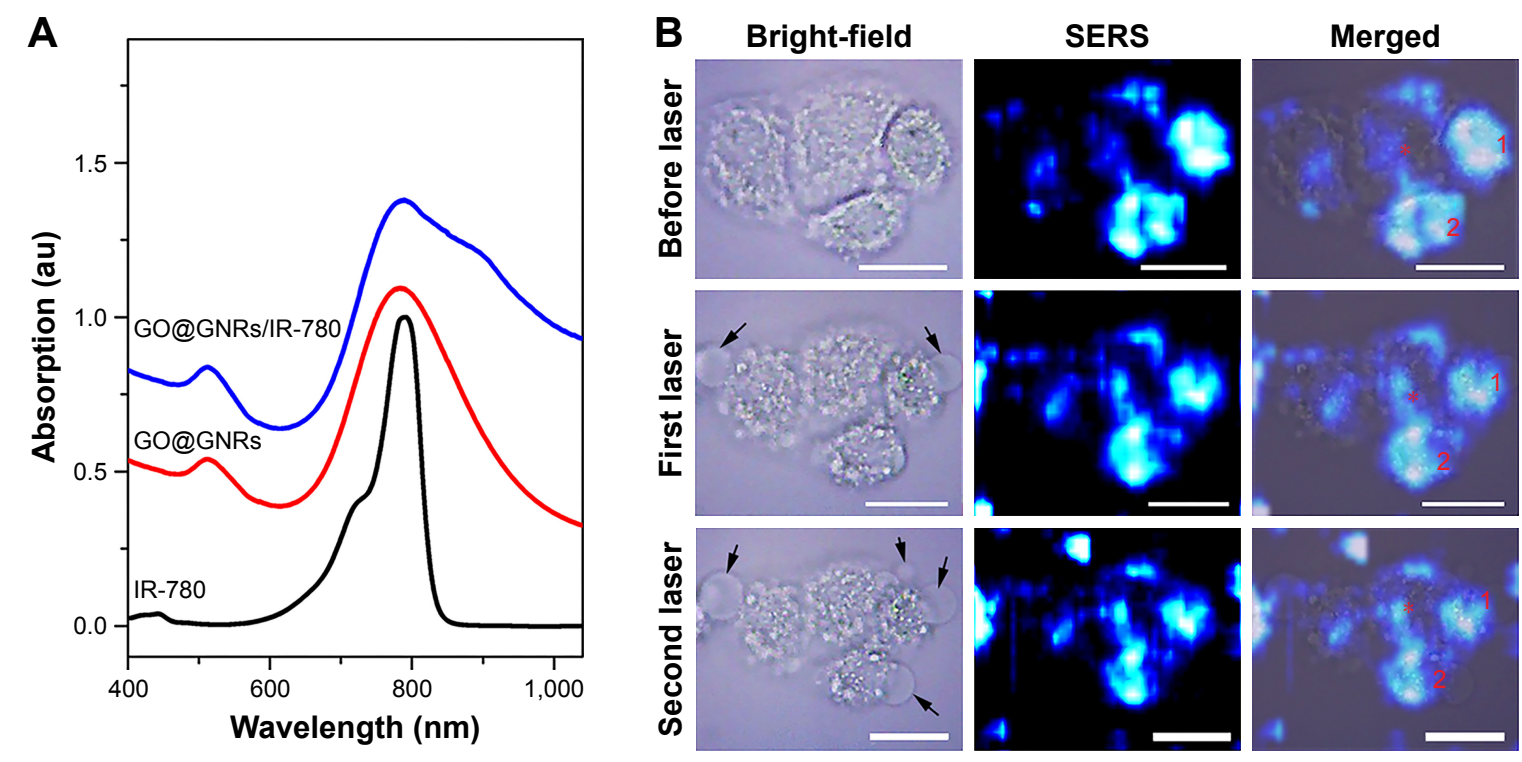

Figure 10 (Continued) 

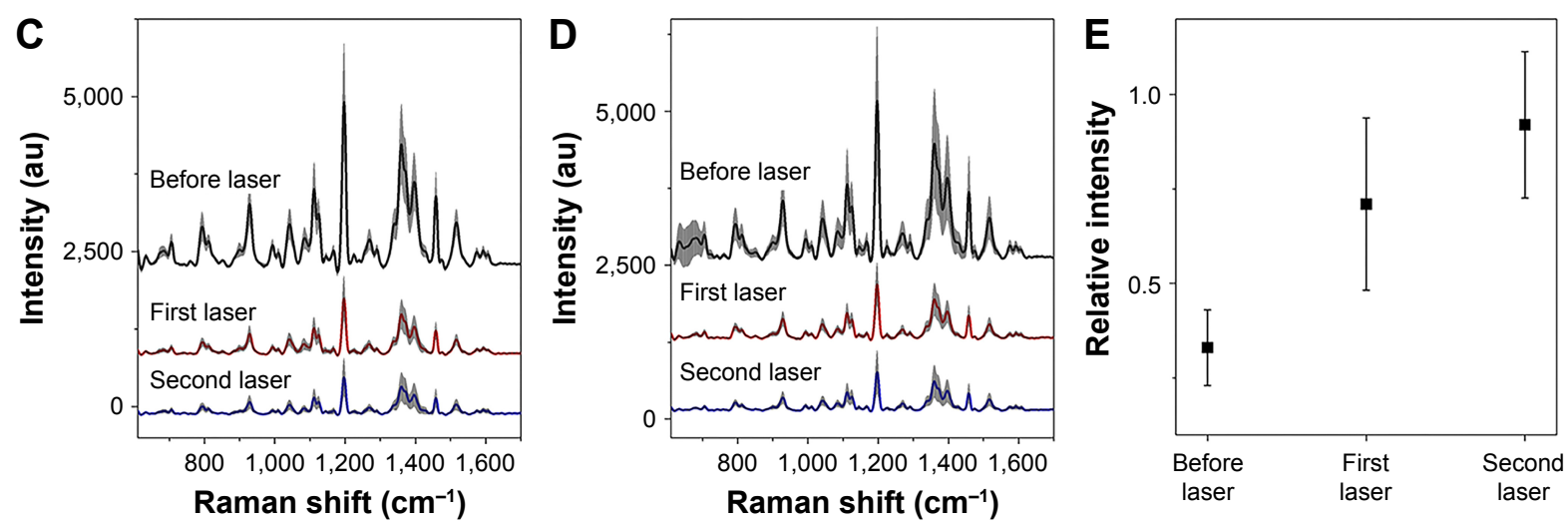

Figure 10 Real-time SERS monitoring of the photothermal ablation effects of the NIR SERS probes.

Notes: (A) UV-Vis-NIR absorbance spectra of NIR SERS probes show the dramatic NIR optical absorbance. (B) NIR SERS bioimaging of KB cells containing SERS probes before and after laser irradiation. The arrows indicate membrane blebbing. *Asterisks indicate typical intracellular area with significant change in the SERS signal. I and 2 indicate two single cancer cells. Scale bars: $20 \mu \mathrm{m}$. (C and D) show the mean SERS spectra of cell I and cell 2 before and after laser irradiation, respectively. The gray area shows the standard deviation. (E) SERS signals the intensity change of asterisk area relative to the overall cell.

Abbreviations: GO, graphene oxide; GNR, gold nanorod; NIR, near-infrared; SERS, surface-enhanced Raman scattering.

with the bare GNRs, GO@GNRs exhibit improved biocompatibility and enhanced NIR SERS activity. Consequentially, the ultrafast NIR SERS imaging based on this type of Raman probe has been accomplished in various living cells. GO@GNRs can also serve as a general nanoplatform to assemble with different dye molecules for fabricating versatile NIR SERS probes, indicating the potential for multi-color NIR SERS bioimaging. Finally, real-time SERS monitoring of photothermal ablation of cancer cells based on GO@GNRs is achieved in vitro. The ultrafast NIR bioimaging capability of GO@GNRs and their theranostic applications open new opportunities for imaging-guided cancer therapy.

\section{Acknowledgments}

This work was supported by the National Natural Science Foundation of China (grants 61335011, 21505047, 61275187, and 61675072), and the Natural Science Foundation of Guangdong Province of China (grants 2014A030310306 and 2014A030311024), the Thousand Talents Plan for Young Professionals, Guangdong Innovative and Entrepreneurial Research Team Program (grant 2013S086), Guangdong Natural Science Foundation (grant 2014A030312018), and Science and Technology Planning Project of Guangdong Province (grant 2016A010103015).

\section{Disclosure}

The authors report no conflicts of interest in this work.

\section{References}

1. Laing S, Gracie K, Faulds K. Multiplex in vitro detection using SERS Chem Soc Rev. 2016;45(7):1901-1918.
2. Xu L-J, Lei Z-C, Li J, Zong C, Yang CJ, Ren B. Label-free surfaceenhanced Raman spectroscopy detection of DNA with single-base sensitivity. J Am Chem Soc. 2015;137(15):5149-5154.

3. Huefner A, Kuan WL, Muller KH, Skepper JN, Barker RA, Mahajan S. Characterization and visualization of vesicles in the endo-lysosomal pathway with surface-enhanced Raman spectroscopy and chemometrics. ACS Nano. 2016;10(1):307-316.

4. Liu Z, Ye B, Jin M, et al. Dye-free near-infrared surface-enhanced Raman scattering nanoprobes for bioimaging and high-performance photothermal cancer therapy. Nanoscale. 2015;7(15):6754-6761.

5. Kurzątkowska K, Santiago T, Hepel M. Plasmonic nanocarrier gridenhanced Raman sensor for studies of anticancer drug delivery. Biosens Bioelectron. 2017;91:780-787.

6. Ilkhani H, Hughes T, Li J, Zhong CJ, Hepel M. Nanostructured SERSelectrochemical biosensors for testing of anticancer drug interactions with DNA. Biosens Bioelectron. 2016;80:257-264.

7. Chen Y, Bai X, Su L, et al. Combined labelled and label-free SERS probes for triplex three-dimensional cellular imaging. Sci Rep. 2016;6: 19173

8. Tian L, Tadepalli S, Fei M, Morrissey JJ, Kharasch ED, Singamaneni S. Off-Resonant Gold Superstructures as Ultrabright Minimally Invasive Surface-Enhanced Raman Scattering (SERS) Probes. Chem Mater. 2015; 27(16):5678-5684.

9. Harmsen S, Huang R, Wall MA, et al. Surface-enhanced resonance Raman scattering nanostars for high-precision cancer imaging. Sci Transl Med. 2015;7(271):271ra277.

10. Iacono P, Karabeber H, Kircher MF. A "schizophotonic" all-in-one nanoparticle coating for multiplexed SE(R)RS biomedical imaging. Angew Chemie. 2014;53(44):11756-11761.

11. Xu Q, Liu W, Li L, Zhou F, Zhou J, Tian Y. Ratiometric SERS imaging and selective biosensing of nitric oxide in live cells based on trisoctahedral gold nanostructures. Chem Commun (Camb). 2017; 53(11):1880-1883.

12. Li JF, Huang YF, Ding Y, et al. Shell-isolated nanoparticle-enhanced Raman spectroscopy. Nature. 2010;464(7287):392-395.

13. Li JF, Anema JR, Wandlowski T, Tian ZQ. Dielectric shell isolated and graphene shell isolated nanoparticle enhanced Raman spectroscopies and their applications. Chem Soc Rev. 2015;44(23):8399-8409.

14. Bian X, Song Z-L, Qian Y, et al. Fabrication of graphene-isolatedAu-nanocrystal nanostructures for multimodal cell imaging and photothermal-enhanced chemotherapy. Sci Rep. 2014;4:6093.

15. Xu W, Mao N, Zhang J. Graphene: a platform for surface-enhanced Raman spectroscopy. Small. 2013;9(8):1206-1224. 
16. Zhang Z, Liu Q, Gao D, et al. Graphene oxide as a multifunctional platform for Raman and fluorescence imaging of cells. Small. 2015; 11(25):3000-3005.

17. Jalani G, Cerruti M. Nano graphene oxide-wrapped gold nanostars as ultrasensitive and stable SERS nanoprobes. Nanoscale. 2015;7(22): 9990-9997.

18. Luo P, Li C, Shi G. Synthesis of gold@carbon dots composite nanoparticles for surface enhanced Raman scattering. Phys Chem Chem Phys. 2012; 14(20):7360-7366.

19. Moon H, Kumar D, Kim H, et al. Amplified photoacoustic performance and enhanced photothermal stability of reduced graphene oxide coated gold nanorods for sensitive photoacoustic imaging. ACS Nano. 2015;9(3):2711-2719.

20. Xu C, Yang D, Mei L, et al. Encapsulating gold nanoparticles or nanorods in graphene oxide shells as a novel gene vector. ACS Appl Mater Interfaces. 2013;5(7):2715-2724.

21. Yim D, Kang H, Jeon SJ, et al. Graphene oxide-encoded Ag nanoshells with single-particle detection sensitivity towards cancer cell imaging based on SERRS. Analyst. 2015;140(10):3362-3367.

22. Kim YK, Na HK, Kim S, Jang H, Chang SJ, Min DH. One-pot synthesis of multifunctional au@graphene oxide nanocolloid core@shell nanoparticles for Raman bioimaging, photothermal, and photodynamic therapy. Small. 2015;11(21):2527-2535.

23. Deng L, Li Q, Yang Y, et al. "Two-step" Raman imaging technique to guide chemo-photothermal cancer therapy. Chemistry. 2015;21(48): 17274-17281.

24. Turcheniuk K, Hage C-H, Spadavecchia J, et al. Plasmonic photothermal destruction of uropathogenic E. coli with reduced graphene oxide and core/shell nanocomposites of gold nanorods/reduced graphene oxide. J Mater Chem B. 2015;3(3):375-386.

25. Xu C, Yang D, Mei L, Li Q, Zhu H, Wang T. Targeting chemophotothermal therapy of hepatoma by gold nanorods/graphene oxide core/shell nanocomposites. ACS Appl Mater Interfaces. 2013;5(24): 12911-12920.

26. Pandey S, Thakur M, Mewada A, Anjarlekar D, Mishra N, Sharon M. Carbon dots functionalized gold nanorod mediated delivery of doxorubicin: tri-functional nano-worms for drug delivery, photothermal therapy and bioimaging. J Mater Chem B. 2013;1(38):4972-4982.
27. Ali MR, Snyder B, El-Sayed MA. Synthesis and optical properties of small Au nanorods using a seedless growth technique. Langmuir. 2012; 28(25):9807-9815.

28. Dong Y, Shao J, Chen C, et al. Blue luminescent graphene quantum dots and graphene oxide prepared by tuning the carbonization degree of citric acid. Carbon. 2012;50(12):4738-4743.

29. Zong S, Wang Z, Yang J, Cui Y. Intracellular $\mathrm{pH}$ sensing using p-aminothiophenol functionalized gold nanorods with low cytotoxicity. Anal Chem. 2011;83(11):4178-4183.

30. Liu Z, Guo Z, Zhong H, Qin X, Wan M, Yang B. Graphene oxide based surface-enhanced Raman scattering probes for cancer cell imaging. Phys Chem Chem Phys. 2013;15(8):2961-2966.

31. Wang X, Wang C, Cheng L, Lee S-T, Liu Z. Noble metal coated single-walled carbon nanotubes for applications in surface enhanced Raman scattering imaging and photothermal therapy. J Am Chem Soc. 2012;134(17):7414-7422.

32. Zavaleta C, de la Zerda A, Liu Z, et al. Noninvasive Raman spectroscopy in living mice for evaluation of tumor targeting with carbon nanotubes. Nano Lett. 2008;8(9):2800-2805.

33. Campion A, Kambhampati P. Surface-enhanced Raman scattering. Chem Soc Rev. 1998;27(4):241-250.

34. McFarland AD, Young MA, Dieringer JA, Van Duyne RP. Wavelengthscanned surface-enhanced Raman excitation spectroscopy. J Phys Chem B. 2005;109(22):11279-11285.

35. Liu Y, Hu Y, Zhang J. Few-layer graphene-encapsulated metal nanoparticles for surface-enhanced Raman spectroscopy. J Phys Chem C. 2014;118(17):8993-8998

36. Ren W, Fang Y, Wang E. A Binary functional substrate for enrichment and ultrasensitive SERS spectroscopic detection of folic acid using graphene oxide/Ag nanoparticle hybrids. ACS Nano. 2011;5(8): 6425-6433.

37. Tong L, Zhao Y, Huff TB, Hansen MN, Wei A, Cheng JX. Gold nanorods mediate tumor cell death by compromising membrane integrity. Adv Mater. 2007;19:3136-3141.
International Journal of Nanomedicine

\section{Publish your work in this journal}

The International Journal of Nanomedicine is an international, peerreviewed journal focusing on the application of nanotechnology in diagnostics, therapeutics, and drug delivery systems throughout the biomedical field. This journal is indexed on PubMed Central, MedLine, CAS, SciSearch $®$, Current Contents ${ }^{\circledR} /$ Clinical Medicine,

\section{Dovepress}

Journal Citation Reports/Science Edition, EMBase, Scopus and the Elsevier Bibliographic databases. The manuscript management system is completely online and includes a very quick and fair peer-review system, which is all easy to use. Visit http://www.dovepress.com/ testimonials.php to read real quotes from published authors. 